\title{
Demonstration of the operation principles of Intermediate Band Solar Cells at room temperature
}

\author{
UPM: E.López ${ }^{\mathrm{a}}$, A.Datas ${ }^{\mathrm{a}}$, I.Ramiro ${ }^{\mathrm{a}}$, P.G. Linares ${ }^{\mathrm{a}}$, E. Antolín ${ }^{\mathrm{a}}$, I. Artacho ${ }^{\mathrm{a}}$, A.Martí ${ }^{\mathrm{a}}$ and A. Luque \\ RCAST: Y. Shoji, T. Sogabe, A. Ogura and Y. Okada ${ }^{b}$ \\ ${ }^{a}$ Instituto de Energía Solar - Universidad Politécnica de Madrid ETSI Telecomunicación, \\ Ciudad Universitaria sn 28040 Madrid, Spain \\ ${ }^{b}$ Research Center for Advanced Science and Technology (RCAST), The University of Tokyo, \\ 4-6-1 Komaba, Meguro-ku, Tokyo 153-8904, Japan
}

\begin{abstract}
In this work we report, for the first time at room temperature, experimental results that prove, simultaneously in the same device, the two main physical principles involved in the operation of intermediate band solar cells: (1) the production of sub-bandgap photocurrent by two optical transitions through the intermediate band; (2) the generation of an output voltage which is not limited by the photon energy absorption threshold. These principles, which had always required cryogenic temperatures to be evidenced all together, are now demonstrated at room temperature on an intermediate band solar cell based on InAs quantum dots with $\mathrm{Al}_{0.3} \mathrm{Ga}_{0.7} \mathrm{As}$ barriers.
\end{abstract}

\section{Introduction}

The structure of an intermediate band solar cell (IBSC) enables an increase of the photo-generated current, as a consequence of the reduction of the photon absorption energy threshold, without the output voltage of the cell being limited by this threshold. This break in the trade-off between current and voltage makes the efficiency limit of an IBSC surpasses the Shockley-Queisser (SQ) limit established for single gap solar cells $\left(63.2 \%\right.$ vs $\left.40.7 \%{ }^{[1]}\right)$.

In IBSCs an electron-hole pair is generated by two mechanisms: the absorption of one photon whose energy is higher than $E_{\mathrm{G}}$ (arrow 3 in Fig. 1), and the absorption of two sub-bandgap photons whose energies are higher than $E_{\mathrm{H}}(\operatorname{arrow}$ 1) and $E_{\mathrm{L}}$ (arrow 2) via two pumping-processes through the IB. The energy reference within the IB for defining $E_{\mathrm{L}}$ and $E_{\mathrm{H}}$ is assumed to be at the center of the intermediate band. On the other hand, in devices implemented with quantum dots (QDs) as it will be our case, the IB is considered to emerge from the fundamental energy level associated to the confined electrons and expected to be narrow and even reduced to a degenerated energy level if QDs are far apart. The isolation of the IB from the metal contacts through the emitters implies that the output voltage is proportional to the difference between the quasi-Fermi level of electrons and the quasi-Fermi level of holes, $E_{\mathrm{FC}}$ and $E_{\mathrm{FV}}$ respectively. Hence, as in a conventional solar cell, the open-circuit voltage $\left(V_{\mathrm{OC}}\right)$ remains limited by the bandgap of the host semiconductor, $E_{\mathrm{G}}$.

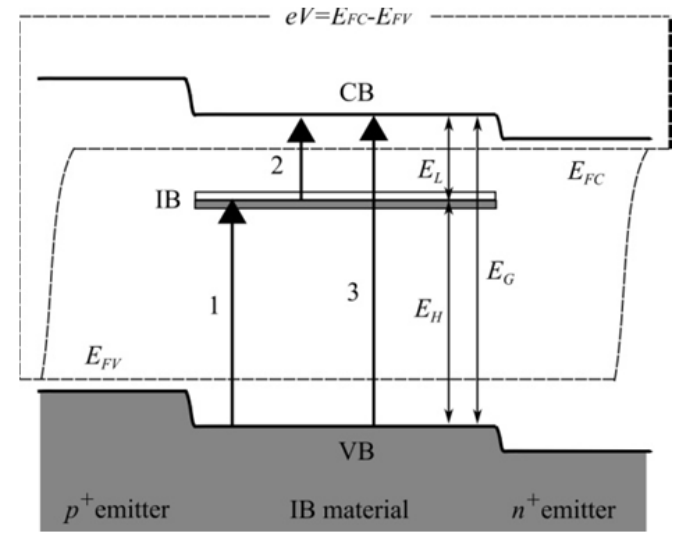

*esther.lopez@ies-def.upm.es / Phone: +34 914533556 / FAX: +34 915446341

(C) 2015. This manuscript version is made available under the Elsevier user license http://www.elsevier.com/open-access/userlicense/1.0/ 
Fig. 1. Band diagram of an IBSC under operating conditions (illumination and forward bias). The three absorption processes and the relationship between the output voltage, $V$, and the quasi-Fermi levels of electrons and holes, $E_{F C}$ and $E_{F V}$, are shown.

Quantum dots (QDs) are one of the approaches used to implement IBSCs because the energy states of the electrons confined in the dots can lead to the formation of the $\mathrm{IB}^{[2]}$. The IBSC operating principles have already been demonstrated in InAs/GaAs QDs ${ }^{[3],[4],[5]}$. Unfortunately, this material is not the best candidate to implement a high efficiency IBSC because of the GaAs low bandgap, $\left(\sim 1.42 \mathrm{eV}\right.$, to be compared to the optimum value, $\left.1.9 \mathrm{eV}^{[1]}\right)$. Furthermore, in this material, it has been obtained experimentally a low value for $E_{\mathrm{L}}$, which leads to undesired thermal escape of electrons between the IB and the $\mathrm{CB}$ at room temperature. Thus, there is interest in shifting towards barrier materials with higher bandgaps ${ }^{[6]}$ and higher $E_{\mathrm{L}}$.

In this respect, IBSCs based on InAs/AlGaAs QDs have been considered as good candidates. This QD system has been studied, providing encouraging results such as a larger $E_{\mathrm{L}}{ }^{6]}$, the generation of two-photon below bandgap photocurrent and voltage-up conversion of low-energy photons ${ }^{[7]}$. Unfortunately, the use of low temperature has still been required in order to decrease thermal escape and obtain some of these results.

In order to work towards the demonstration of the IBSC operating principles at RT, we have manufactured InAs QDs based on AlGaAs barriers with a concentration of aluminum slightly larger than the one used in the works cited above (Al content of 30\% instead of 25\%). These cells have been characterized by photocurrent measurements and currentvoltage curves under concentrated white-light illumination and the results will be shown in following sections.

\section{Material and methods}

\subsection{InGaAs/AlGaAs solar cell}

The solar cells studied in this work were grown on $350 \mu \mathrm{m}$ thick $\mathrm{n}^{+}$-GaAs substrates by molecular beam epitaxy (MBE) at the University of Tokyo. The layer structure of these samples is shown in Fig. 2. Two different types of samples were manufactured: a sample with 25 layers of $\mathrm{InGaAs} / \mathrm{Al}_{0.3} \mathrm{Ga}_{0.7} \mathrm{As}$ QDs in the region labeled as "Middle", and a reference sample without QDs in which this middle region consists of $\mathrm{Al}_{0.3} \mathrm{Ga}_{0.7} \mathrm{As}$. In both samples this layer has no intentional doping and is sandwiched between conventional $\mathrm{p}\left(2 \times 10^{18} \mathrm{~cm}^{-3}\right)$ and $\mathrm{n}\left(8 \times 10^{16} \mathrm{~cm}^{-3}\right)$ type $\mathrm{Al}_{0.3} \mathrm{Ga}_{0.7} \mathrm{As}$.

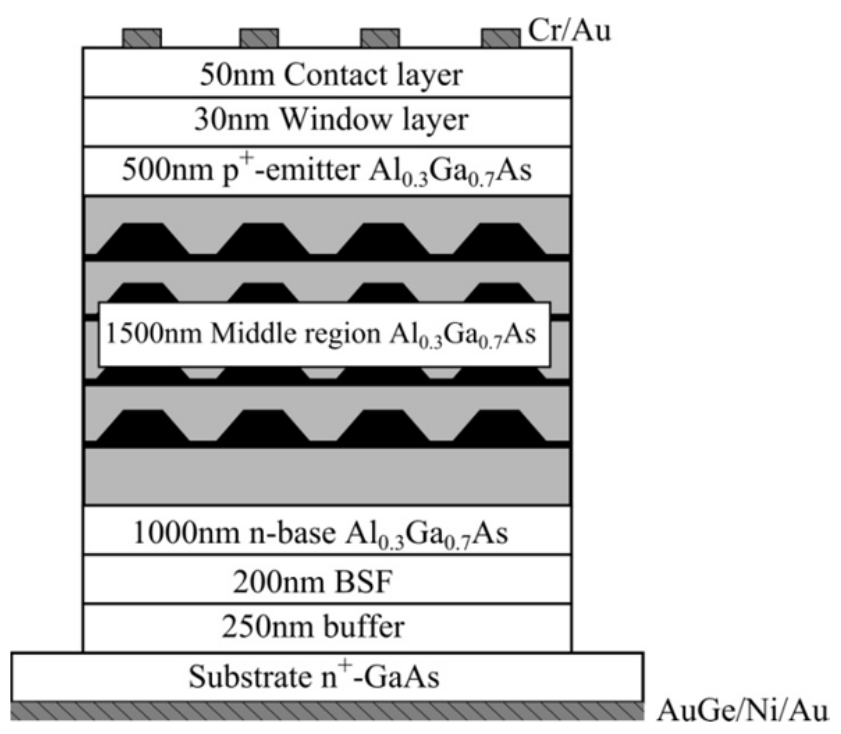

Fig. 2. Layer structure of the QDs solar cell studied in this work. The middle region consists of 25 layers of $\mathrm{InGaAs} / \mathrm{Al}_{0.3} \mathrm{Ga}_{0.7} \mathrm{As}$ QDs spaced $60 \mathrm{~nm}$. In the case of the reference cell, the middle region consists of $\mathrm{Al}_{0.3} \mathrm{Ga}_{0.7} \mathrm{As}$ with no QDs.

A back surface field layer $\left(\mathrm{Al}_{0.75} \mathrm{Ga}_{0.25} \mathrm{As} \mathrm{n}\right.$-type doped $\left.1 \times 10^{17} \mathrm{~cm}^{-3}\right)$ was grown on top of a $250 \mathrm{~nm} \mathrm{n}^{+}$-GaAs buffer layer $\left(1 \times 10^{18} \mathrm{~cm}^{-3}\right)$. A window layer $\left(\mathrm{Al}_{0.75} \mathrm{Ga}_{0.25}\right.$ As p-type doped $\left.5 \times 10^{18} \mathrm{~cm}^{-3}\right)$ was grown on top of the emitter, 
followed by a $\mathrm{p}^{+}$-GaAs $\left(2 \times 10^{19} \mathrm{~cm}^{-3}\right)$ contact layer. Finally, metal contacts were deposited by thermal evaporation both on the back $(\mathrm{AuGe} / \mathrm{Ni} / \mathrm{Au})$ and the front $(\mathrm{Cr} / \mathrm{Au})$ sides of the device.

InGaAs QDs were fabricated by the growth interruption technique. Hence, the QDs were formed during the growth interruption after 10.5 MLs of InGaAs were deposited, at high growth rate of $\sim 1 \mathrm{ML} / \mathrm{s}$, on intrinsic $\mathrm{Al}_{0.3} \mathrm{Ga}_{0.7} \mathrm{As}_{\mathrm{spacers}}$ of $60 \mathrm{~nm}^{[8,9]}$. Due to the doping level and the thickness of the layers in Fig. 2, the QDs were located inside the spacecharge region of the solar cell. Hence, as described in [10], in equilibrium, it is warranted that some QD layers are partially filled with electrons at the prize that some other layers are either filled or emptied of electrons.

\subsection{Photocurrent measurements}

The photocurrent measurements are performed at RT by illuminating the samples with sub-bandgap photons with energy in the range from $0.2 \mathrm{eV}$ to $1.4 \mathrm{eV}$ (the $\mathrm{Al}_{0.3} \mathrm{Ga}_{0.7} \mathrm{As}$ bandgap is around $1.8 \mathrm{eV}^{[11]}$ ). Therefore, the photocurrent must be generated by optical transitions that involve intermediate levels, which exist, in principle, only in the solar cells with QDs. Hereinafter, we will refer to these levels as IB.

The measured photocurrent responds to the monochromatic light that comes from the output of a $1 / 4 \mathrm{~m}$ monochromator. Inside the monochromator, the light of a $100 \mathrm{~W}$ halogen lamp is diffracted and, at the output, appropriate optical filters are placed to guarantee the monochromaticity of the photon beam. In order to maximize the measured photocurrent, the output monochromatic beam is concentrated as much as possible with a parabolic mirror, guaranteeing that the light spot covers the total area of the solar cells $\left(0.032 \mathrm{~cm}^{2}\right)$. The spectral photon flux is in the range of $4.3 \times 10^{14}-3.7 \times 10^{20} \mathrm{~m}^{-2} \cdot \mathrm{s}^{-1} \cdot \mathrm{nm}^{-1}$ for photons with energy above $0.9 \mathrm{eV}$ and is measured for each energy with a calibrated Newport photodetector. This calibration also allows measuring the external quantum efficiency of the cell (EQE) for photon energies above $0.9 \mathrm{eV}$. It can be assumed that for energies lower than $0.9 \mathrm{eV}$ the photon flux that illuminates the solar cells is lower than the values of this range but no absolute calibration has been possible.

The intensity of the monochromatic beam is modulated at $33 \mathrm{~Hz}$ using an optical chopper, and the alternate photocurrent generated by the cell in response to this illumination is measured with a lock-in amplifier. For each of the two samples studied in this work, this photocurrent is measured under two different operation conditions, to know, with and without illumination by means of a continuous-wave $(\mathrm{cw})$ laser diode of $1.32 \mathrm{eV}$ (energy lower than $E_{G}$ ). The latter illumination alone produces a continuous photocurrent density of $0.52 \mathrm{~mA} / \mathrm{cm}^{2}$ in the QD solar cell. This photocurrent density is equivalent to the current density generated by this solar cell when illuminated by 0.5 suns (resulting from integrating the EQE of the cell for photons with energy higher than $0.9 \mathrm{eV}$ with ASTM-G173-0 spectrum). During all photocurrent measurements, samples were biased at $0 \mathrm{~V}$.

\subsection{Concentration measurements}

The short-circuit current density $\left(J_{\mathrm{SC}}\right)$ vs. $V_{\mathrm{OC}}$ curves under different levels of illumination are measured at RT using the experimental technique described in [12]. In this technique, the light of a Metz Mecablitz 54 MZ-3 xenon flash lamp is concentrated by a $\mathrm{CaF}_{2}$ lens to illuminate the cells with different irradiances during the time of the flash discharge. This time is approximately $20 \mathrm{~ms}$ long, although $90 \%$ of the energy is discharged approximately within the first $5 \mathrm{~ms}$. Two flash pulses are used to obtain the $J_{\mathrm{SC}}-V_{\mathrm{OC}}$ pairs. During the first pulse, samples are biased at $0 \mathrm{~V}$ using a 4-wire Keithley source meter, so that $J_{\mathrm{SC}}$ values at different irradiances are obtained as the intensity of the light of the flash decays. During the second pulse, samples operate at open-circuit, so that $V_{\mathrm{OC}}$ values at different irradiances are obtained. Both sets of data, $J_{\mathrm{SC}}$ and $V_{\mathrm{OC}}$, are acquired with a sampling rate of $200 \mathrm{kHz}$ by the analog inputs of a National Instrument PCI DAQ card. This DAQ system only can measure voltage signals, so that, in order to convert $J_{\mathrm{SC}}$ into a measurable voltage signal, the use of a calibrated shunt is required. The correlation between these two sets of data is obtained by a silicon reference detector, which is placed close to the sample during the measurements. The illumination level is estimated by dividing the measured photogenerated-current density $\left(J_{\mathrm{L}}\right)$, which is assumed proportional to the irradiance level, by the theoretical value of the photogenerated-current density at one sun (for the QD solar cell, this current density is $1.05 \mathrm{~mA} / \mathrm{cm}^{2}$ under ASTM-G173-0 spectrum).

\section{Results}

\subsection{Two-photon below bandgap absorption at room temperature}


For comparison, the measured photocurrents for the sample with QDs and for the reference sample are plotted in Fig. 3. These measurements reveal the generation of a notable sub-bandgap photocurrent in the QD solar cell (dashed red line) which increases, when the sample is also illuminated with the $\mathrm{cw}$ laser diode of $1.32 \mathrm{eV}$ (solid red line), in the range from $0.3 \mathrm{eV}$ to $1.17 \mathrm{eV}$. Under these conditions, photocurrent is produced using the far infrared (IR) down to $0.3 \mathrm{eV}$. There is an abrupt change in photocurrent around $1.17 \mathrm{eV}$. This photon energy corresponds to the sub-bandgap $\mathrm{E}_{\mathrm{H}}$ as we shall see in the discussion.

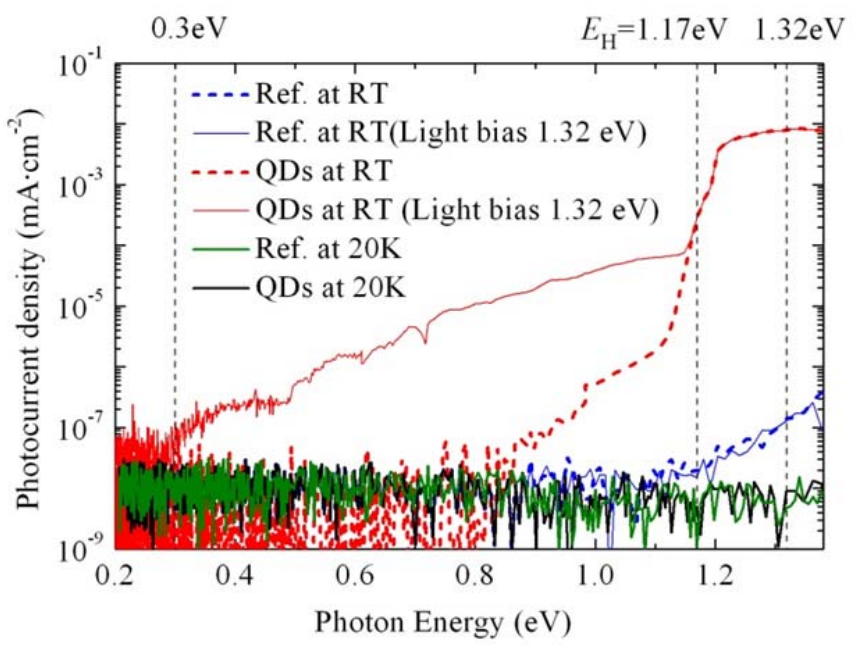

Fig. 3. Sub-bandgap photocurrent density at room temperature from the reference cell (blue lines) and the QD solar cell (red lines). The solid lines are the photocurrent density extracted when the light of a laser of energy $1.32 \mathrm{eV}$ biases the samples. In the case of the QD solar cell this light bias produces a photocurrent density of $0.52 \mathrm{~mA} / \mathrm{cm}^{2}$, equivalent to the illumination of 0.5 suns. The position of the IB is identified at $1.17 \mathrm{eV}$ from the VB and the absorption threshold for the sample with QDs is $0.3 \mathrm{eV}$. Photocurrents at $20 \mathrm{~K}$ are plotted for comparison (green and black lines).

An unexpected photocurrent appears in the reference sample when illuminated with photons of energies from $1.2 \mathrm{eV}$ to $1.4 \mathrm{eV}$ (dashed blue line). In this case, however, the presence of the $1.32 \mathrm{eV}$ light bias does not change the photocurrent curve (solid blue line). This measured photocurrent could be related to the presence of intermediate levels (resulted from native defects in the $\mathrm{Al}_{0.3} \mathrm{Ga}_{0.7} \mathrm{As}^{[13]}$ ) which do not perform as an IB (there is no response to two-photon subbandgap illumination). Similar results have been obtained in other materials ${ }^{[14],[15]}$.

\subsection{Open-circuit voltage surpasses the absorption threshold}

Fig. 4 shows that the $\mathrm{V}_{\mathrm{OC}}$ produced by the QD solar cell at RT covers the range from $0.73 \mathrm{~V}$ to $1.07 \mathrm{~V}$ when it is illuminated by a Xenon lamp with irradiance levels from 0.5 suns to $\sim 390$ suns. 


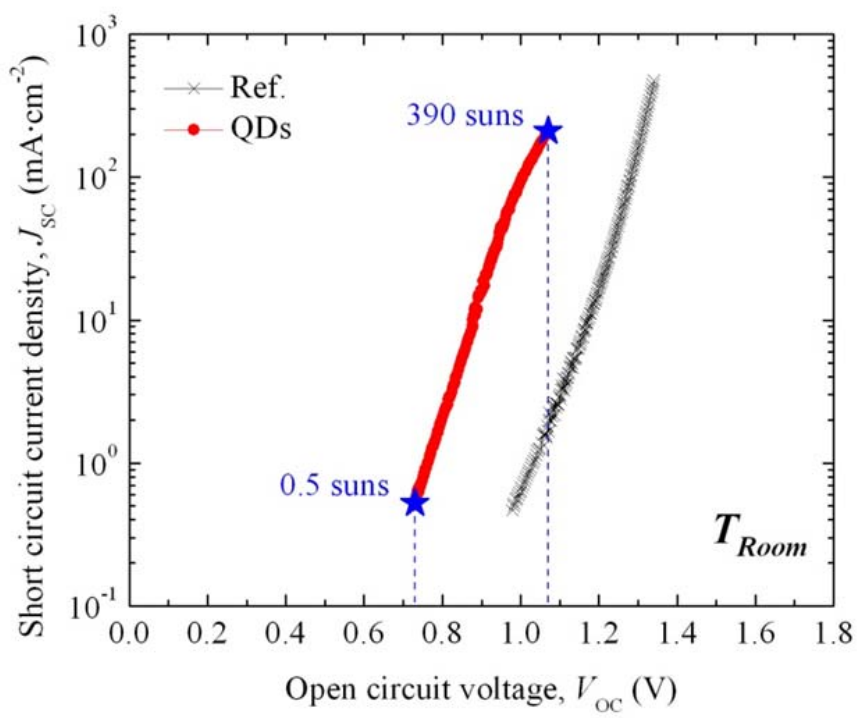

Fig. 4. $J_{\mathrm{SC}}$ vs. $V_{\mathrm{OC}}$ at room temperature for different levels of illumination. The maximum concentration achieved is $\sim 390$ suns. It produces a $V_{\mathrm{OC}}$ of $1.07 \mathrm{~V}$ in the QD solar cell.

\section{Discussion}

In an ideal IBSC, with selective absorption coefficients, it is not possible to extract photocurrent when the solar cell is illuminated with a monochromatic sub-bandgap photon energy beam. The reason is that, in such an ideal cell, photons with a specific energy can only be absorbed in one optical transition. However, the results plotted by the dashed red line in Fig. 3 shows that it is possible to produce photocurrent when the QD solar cell is illuminated only with monochromatic light, whose photon energy varies from $0.9 \mathrm{eV}$ to $1.4 \mathrm{eV}$. Previous works ${ }^{[16],[17]}$ have explained the origin of this sub-bandgap photocurrent as the result of two pumping-processes: optical transitions of electrons from the VB to the IB and a thermal escape processes from the IB to the CB. This thermal escape is undesired because no electrical work can be extracted from it and its thermal origin is identified because this sub-bandgap photocurrent disappears when the temperature decreases. The same results are obtained in the QD solar cell studied in this work. The photocurrent generated when the QD solar cell is illuminated only with monochromatic photon energies from 0.9 $\mathrm{eV}$ to $1.4 \mathrm{eV}$ (dashed red line in Fig. 3) disappears when the temperature decreases down to 20K (black line in Fig. 3). This may be because, once thermal excitation from the IB to the CB has been suppressed, there is no second photon capable of pumping and electron form the IB to the CB. The suppression of sub-bandgap photocurrent at 20K suggests that the absorption coefficients associated to sub-bandgap generation processes (labeled as 1 and 2 in Fig. 1) do not overlap at low temperature since, otherwise, a photon energy (larger than $0.9 \mathrm{eV}$ ) should also be able to pump an electron from the IB to the $\mathrm{CB}$. These absorption coefficients depend on the electron concentration in the IB. In the case of the QD solar cell, we assume that this concentration is determined by the fact that the IB is located inside space-charge region (as discussed in section 2.1) and therefore, it should not change significantly with temperature. According to this assumption, we would expect that absorption coefficients associated to sub-bandgap generation processes do not overlap at room temperature either.

According to this argument, it is possible to identify $E_{\mathrm{H}}$ from the results represented by dashed red line in Fig. 3, as the photon energy at which there is an abrupt change in photocurrent $(\sim 1.17 \mathrm{eV})$. It is more difficult to resolve $E_{\mathrm{L}}$ if we illuminate the solar cell with only monochromatic light, because it is more difficult to pump electrons from the VB to the IB by thermal escape since $E_{\mathrm{H}}$ is larger than $E_{\mathrm{L}}$. Hence, to resolve $E_{\mathrm{L}}$ we need to increase the pumping of electrons from the VB to the IB by illuminating the sample also with photons with energy larger than $E_{\mathrm{H}}{ }^{[18]}$. This is how the results represented by the red line in the Fig. 3 have been obtained. In this case, a cw laser diode of $1.32 \mathrm{eV}$ has been used to illuminate continuously the solar cell, producing a continuous photocurrent equivalent to 0.5 suns illumination. 
Under these conditions, we have identified the two sub-bandgaps, $E_{\mathrm{L}} \approx 0.3 \mathrm{eV}$ and $E_{\mathrm{H}}=1.17 \mathrm{eV}$. Therefore, taking into account that $E_{\mathrm{G}}$ is around $1.8 \mathrm{eV}^{[11]}$, it is possible to infer a VB QD offset of around $0.33 \mathrm{eV}$.

These results have been obtained because of the presence of two sub-bandgap transitions, between the VB and the IB, and between the IB and the CB. Previous works have reported the presence of these transitions at RT in QD-IBSCs ${ }^{[19]}$. However, up to now, low temperature has always been required to spectrally resolve them ${ }^{[7]}$. In Fig. 3 we present, for the first time at RT, the spectral resolution of both sub-bandgap transitions (solid red line).

The upgrades carried out in this work to reach this result are based on improvements of the experimental setup and the manufacturing of QDs based on barriers with a concentration of aluminum larger than those used in previous works (Al content of $30 \%$ instead of $25 \%$ ). The setup's improvements have been aimed to increase the intensity of the monochromatic light that illuminates the samples by two orders of magnitude approximately. The increase in the aluminum concentration has been aimed to reduce the thermal escape by enlarging the bandgap $E_{\mathrm{G}}$. Notice that, in spite of using larger values for $E_{\mathrm{G}}$, thermal escape still has a significant role in the performance of the device and therefore this QD solar cell still does not operate as an ideal IBSC, capable of significantly increasing its efficiency over single gap solar cells

These two strategies have favored the detection of the lowest absorption threshold measured at RT in a QD-IBSC. The absorption threshold is $0.3 \mathrm{eV}$ and corresponds to $E_{\mathrm{L}}$. At this temperature, to illuminate the samples with an irradiance of 0.5 suns results in a $V_{\mathrm{OC}}$ of $0.73 \mathrm{~V}$. Therefore, the $V_{\mathrm{OC}}$ surpasses the absorption threshold at RT, for the first time without the use of concentrated illumination. In order to the efficiency of the QD solar cell surpass the SQ limit, it is necessary, however, that the $V_{\mathrm{OC}}$ surpasses both sub-bandgaps $\left(E_{\mathrm{H}}\right.$ and $\left.E_{\mathrm{L}}\right)$, which is not the case.

An important observation is that, the two operation principles of an IBSC have been proven under illumination conditions which imply similar photocurrent generations $\left(0.52 \mathrm{~mA} / \mathrm{cm}^{2}\right.$, equivalent to the photocurrent density generated by 0.5 suns).

\section{Conclusions}

The important goal which has been reached in this work is to prove, for the first time at RT, the two operation principles of an IBSC: the sub-bandgap photocurrent production by two optical transitions through the IB, and the generation of an output voltage which is not limited by the absorption threshold. This has been possible because the spectral resolution of $E_{\mathrm{L}}$ has lead to the detection at RT of a low absorption threshold of $0.3 \mathrm{eV}$. These results have been obtained from a solar cell based on InGaAs/ $\mathrm{Al}_{0.3} \mathrm{Ga}_{0.7} \mathrm{As}$ QDs, under operation conditions equivalent to illuminating the IBSC with 0.5 suns.

\section{Acknowledgments}

This work has been supported by the European Commission through the NGCPV Project (Grant Number 283798), the Spanish Ministerio de Economía y Competitividad through the Project Promesa (ENE2012-37804-C02-01) and also by the Programme MADRID PV, funded by the Comunidad de Madrid (Grant number S2013/MAE-2780). EA acknowledges the grant funds coming from L'OREAL-UNESCO "For women in science" Call.2014-2015. This work is also supported by New Energy and Industrial Technology Development Organization (NEDO), and Ministry of Economy, Trade and Industry (METI), Japan. The Strategic International Cooperative Program by Japan Science and technology Agency (JST) is also acknowledged.

\section{References}

[1] A. Luque and A. Martí, "Increasing the efficiency of ideal solar cells by photon induced transitions at intermediate levels," Physical Review Letters, vol. 78, pp. 5014-5017, 1997.

[2] A. Martí, L. Cuadra, and A. Luque, "Quantum dot intermediate band solar cell," in 28th IEEE Photovoltaic Specialists Conference, Anchorage (Alaska), 2000, pp. 940-943.

[3] A. Martí, E. Antolín, C. R. Stanley, C. D. Farmer, N. López, P. Díaz, et al., "Production of photocurrent due to intermediate-to-conduction-band transitions: a demonstration of a key operating principle of the intermediate-band solar cell," Physical Review Letters, vol. 97, p. 247701, 2006.

[4] P. García-Linares, A. Martí, E. Antolín, C. D. Farmer, I. Ramiro, C. R. Stanley, et al., "Voltage recovery in intermediate band solar cells," Solar Energy Materials and Solar Cells, vol. 98, pp. 240-244, 2012. 
[5] Y. Shoji, K. Akimoto, and Y. Okada, "Self-organized InGaAs/GaAs quantum dot arrays for use in highefficiency intermediate-band solar cells," Journal of Physics D-Applied Physics, vol. 46, Jan 16.

[6] I. Ramiro, E. Antolin, M. J. Steer, P. G. Linares, E. Hernandez, I. Artacho, et al., "InAs/AlGaAs quantum dot intermediate band solar cells with enlarged sub-bandgaps," in 38th IEEE Photovoltaic Specialists Conference, Austin (TE) EEUU, 2012, pp. 000652-000656.

[7] I. Ramiro, E. Antolin, P. G. Linares, E. Lopez, I. Artacho, A. Datas, et al., "Two-photon photocurrent and voltage up-conversion in a quantum dot intermediate band solar cell," in Photovoltaic Specialist Conference (PVSC), 2014 IEEE 40th, pp. 3251-3253.

[8] K. Akahane and N. Yamamoto, "Formation of InAs quantum dots at ultrahigh growth rates," Physica E: Lowdimensional Systems and Nanostructures, vol. 42, pp. 2735-2738.

[9] S. Takeyoshi, A. Takeru, M. Masahiko, N. Shigeru, and K. Michio, "Highly Stacked and High-Quality Quantum Dots Fabricated by Intermittent Deposition of InGaAs," Japanese Journal of Applied Physics, vol. 49, p. 030211.

[10] A. Marti, E. Antolin, E. Canovas, N. Lopez, P. G. Linares, A. Luque, et al., "Elements of the design and analysis band solar of quantum-dot intermediate cells," Thin Solid Films, vol. 516, pp. 6716-6722, Aug 2008.

[11] M. Levinshtein, S. L. Rumyantsev, and M. Shur, Handbook Series on Semiconductor Parameters: Ternary and quaternary A3B5 semiconductors: World Scientific, 1999.

[12] P. G. Linares, A. Marti, E. Antolin, I. Ramiro, E. Lopez, C. D. Farmer, et al., "Low-Temperature Concentrated Light Characterization Applied to Intermediate Band Solar Cells," Photovoltaics, IEEE Journal of Photovoltaics, vol. 3, pp. 753-761, 2012.

[13] L. Pavesi and M. Guzzi, "PHOTOLUMINESCENCE OF ALXGA1-XAS ALLOYS," Journal of Applied Physics, vol. 75, pp. 4779-4842, May 1994.

[14] H. G. Grimmeiss, W. Kischio, and H. Koelmans, "P-N-JUNCTION PHOTOVOLTAIC EFFECT IN ZINCDOPED GAP," Solid-State Electronics, vol. 5, pp. 155-159, 1962.

[15] D. C. Reynolds, G. Leies, L. L. Antes, and R. E. Marburger, "Photovoltaic Effect in Cadmium Sulfide," Physical Review, vol. 96, pp. 533-534, 1954.

[16] E. Antolin, A. Marti, C. D. Farmer, P. G. Linares, E. Hernandez, A. M. Sanchez, et al., "Reducing carrier escape in the InAs/GaAs quantum dot intermediate band solar cell," Journal of Applied Physics, vol. 108, p. 7, Sep 2010.

[17] G. Jolley, L. Fu, H. F. Lu, H. H. Tan, and C. Jagadish, "The role of intersubband optical transitions on the electrical properties of InGaAs/GaAs quantum dot solar cells," Progress in Photovoltaics, vol. 21, pp. 736746, Jun.

[18] M. C. Wagener, P. J. Carrington, J. R. Botha, and A. Krier, "Evaluation of the two-photon absorption characteristics of $\mathrm{GaSb} / \mathrm{GaAs}$ quantum rings," Journal of Applied Physics, vol. 116, pp. - .

[19] Y. Okada, T. Morioka, K. Yoshida, R. Oshima, Y. Shoji, T. Inoue, et al., "Increase in photocurrent by optical transitions via intermediate quantum states in direct-doped InAs/GaNAs strain-compensated quantum dot solar cell," Journal of Applied Physics, vol. 109, pp. 024301-024301-5. 